\title{
Industry-energy system management by a Copula-based stochastic programming approach
}

\author{
Ying Zhu ${ }^{1, a^{*}}$, Zhao Wei ${ }^{1, b}$, Yexin $\mathrm{Li}^{2, c}$, Jingqi Luo ${ }^{1, \mathrm{~d}}$, Bizhou Ge $\mathrm{Ge}^{1, \mathrm{e}}$ \\ ${ }^{1}$ School of Environment and Municipal Engineering, Xi' an University of Architecture and Technology, Xi'an, China \\ ${ }^{2}$ Ankang Environmental Engineering Design Limited Company, Ankang, ChinaType the author addresses here
}

\begin{abstract}
In this study, a Copula-based stochastic industry-energy system management (CSIE) model was developed based on Copula-based stochastic programming and interval linear programming. CSIE model can not only deal with extreme random events in industry-energy system (IES) of resource-dependent cities, but also quantify the risks of industrial energy demand-supply. To prove the practicability, a case study of IES planning in Yulin city was represented. Reasonable solutions of energy production and industrial energy consumption strategy were obtained, which can guarantee that pollutant emission meets the environmental requirements, and the system cost gets the lowest during 2021-2035. Furthermore, CSIE model could be spread to IES management in similar resource-dependent cities.
\end{abstract}

\section{Introduction}

Industry-energy system (IES) is filled with extreme random events, especially in resource-dependent cities. For example, the extremely unreasonable energy consumption structure, the extremely irrational industrial structure, and the extremely fragile ecological environment [1]. In the context of industrialization and urbanization, sustained industrial production is significant for economic development. However, long-term excessive consumption of energy resources would not only cause immense local energy wastage and exacerbate energy shortage risks, but also inevitably lead to tremendous pollutant emissions, which aggravates the ecological restoration pressure to the fragile ecological environment [2]. Planning IES by optimization model is one of the most effective ways to cope with energy shortage and atmosphere environment pollution, as well as guarantee sustained industrial production. Nevertheless, extreme random events would interfere inversion analysis of IES through traditional models, then reduce the reliability of planning schemes [3]. Therefore, the objective of this paper is to develop a Copula-based stochastic industryenergy system management (CSIE) model for dealing with extreme random uncertainties and supporting IES management of resource-dependent cities, and taking Yulin city, China as an example to verify the practicability of the model.

\section{Application}

\subsection{Overview of the study area}

\footnotetext{
a*email: zhuyingxauat@163.com, bemail: 948149584@qq.com

cemail: 280645040@qq.com demail: 1115185298@qq.com, eemail: gebz@163.com
}

Yulin city $\left(107^{\circ} 28^{\prime}\right.$ to $115^{\circ} 15^{\prime} \mathrm{E}, 36^{\circ} 57^{\prime}$ to $\left.39^{\circ} 34^{\prime} \mathrm{N}\right)$ is situated in the northernmost part of Shaanxi Province, China, which has an administrative area of $42,902.20 \mathrm{~km}^{2}$. By the end of 2020, the population was 3.86 million. Yulin city is a typical resource-dependent city rich in energy resources, especially coal, oil and natural gas, known as "China's Kuwait". According to Yulin Statistical Yearbook (2019), the total energy consumption of the city was 0.14 billion tons of standard coal, of which coal consumption was 0.12 billion tons of standard coal. The GDP of the city was RMB $¥ 413.63$ billion, including RMB $¥ 231.30$ billion which comes from the coal mining and processing industry in late 2019. Yulin city is located in a transition zone from $\mathrm{Mu}$ Us Desert to Loess Plateau, with loessal soil and aeolian soil, accounting for $49.50 \%$ and $27.30 \%$ respectively. The city features semi-arid, arid climatic conditions with an average annual wind speed of $2.90 \mathrm{~m} / \mathrm{s}$ and average annual precipitation of $446.50 \mathrm{~mm}$.

In view of the above information, there are many extreme random events that exist in IES of Yulin city, including: (a) the energy structure is extremely unreasonable in Yulin city, because more than $80 \%$ of its energy consumption is from burning coal; (b) the industrial structure is extremely irrational, as the GDP of coal related industries occupies more than $50 \%$ of the city; (c) due to extremely uneven spatial and temporary distribution of natural conditions, Yulin city shows extremely fragile ecological characteristics. Thus, it is necessary to adjust current energy production, industrial energy consumption structure, and pollutant emission strategy to promote sustainable development of the city on the basis of dealing with extreme random uncertainties. 


\subsection{Data analysis and process}

In this paper, the relevant energy, industrial, technical and economic data were collected from representative reports, government official reports, literature surveys, and expert consultations. Particularly, the energy output data was obtained from Yulin Statistical Yearbook (from 2000 to 2019). The historical data of primary energy output and secondary energy output from years 2000-2019 were used to determine marginal distribution functions and joint cumulative distribution functions. The correlation between the two random variables was tested by Pearson's linear correlation tests [4]. Results indicate good correlation between primary energy output and secondary energy output. Then, through comparing five bivariate Copula functions, Clayton-Copula was selected as the best one for joint cumulative distribution simulation with the minimum values of root mean squared error (RMSE), Akaike information criterion (AIC), and Bayesian information criterion (BIC), as shown in Table 1 [5]. The joint cumulative distribution for primary energy output and secondary energy output using Clayton-Copula is shown in Figure 1.

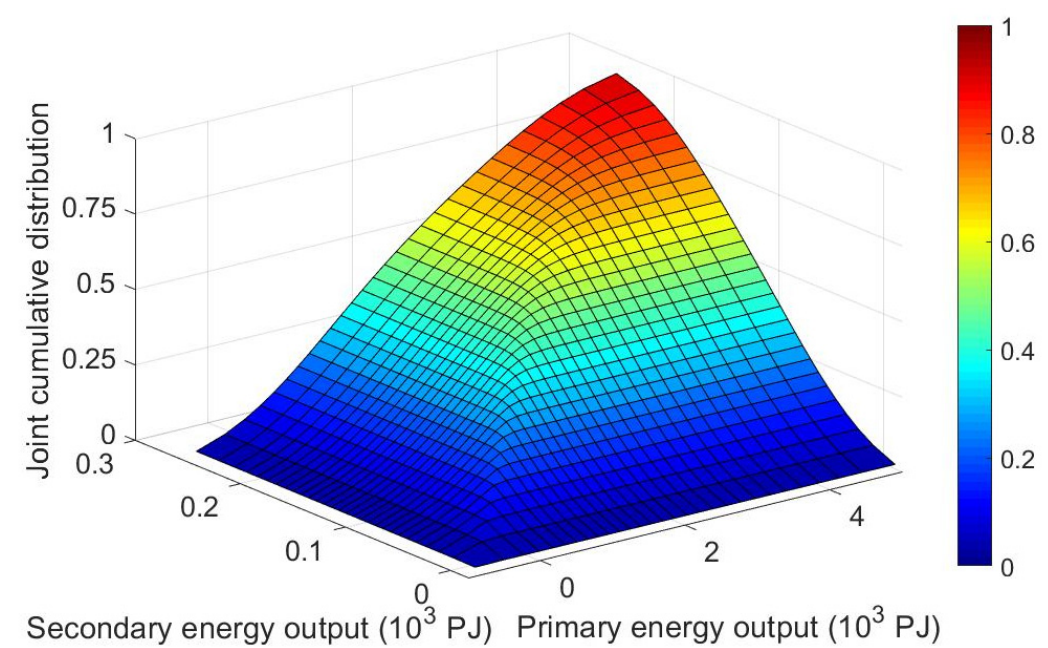

Figure 1. Joint cumulative distribution for primary energy output and secondary energy output using Clayton-Copula

Table 1. Values of parameters and evaluation metric for bivariate Copula functions

\begin{tabular}{|c|c|c|c|c|}
\hline Copula functions & Copula function $C(u, v ; \theta)$ & RMSE & AIC & BIC \\
\hline Gaussian & $\varphi_{\rho}\left(\varphi^{-1}(u), \varphi^{-1}(v)\right)$ & 0.0932 & -64.4294 & -61.1512 \\
\hline $\mathrm{t}$ & $t_{\theta, k}\left(t_{k}^{-1}(u), t_{k}^{-1}(v)\right)$ & 0.0846 & -67.1541 & -63.8760 \\
\hline Gumbel & $\exp \left(-\left((-\ln u)^{\theta}+(-\ln v)^{\theta}\right)^{1 / \theta}\right)$ & 0.0893 & -65.6564 & -62.3783 \\
\hline Clayton & $\left(u^{-\theta}+v^{-\theta}-1\right)^{-1 / \theta}$ & 0.0821 & -68.0028 & -64.7247 \\
\hline Frank & $-1 / \theta \ln \left(1+\left(e^{-\theta u}-1\right)\left(e^{-\theta v}-1\right) /\left(e^{-\theta}-1\right)\right)$ & 0.0828 & -67.7593 & -64.4811 \\
\hline
\end{tabular}

\subsection{Modelling formulation}

A Copula-based stochastic industry-energy system management (CSIE) model could be formulated based on Copula-based stochastic programming and interval linear programming, aiming at system cost minimization, and considering uncertain factors including energy availability, industrial product demand, Gaussian dispersion of pollutant and environmental requirements. The CSIE model has superiority in extreme random uncertainties reflection, and can deal with the tradeoff among energy production, industrial energy consumption strategy, pollutant emission control and system economy.
2.3.1. Objective function. The objective of CSIE model is to minimize the total system cost, which depends on the following equation:

$$
\min f^{ \pm}=\sum\left(C_{G}+C_{Y}+C_{S}+C_{W}+C_{P}\right)
$$

(1) Energy purchase cost:

$$
C_{G}=\sum_{p=1}^{3} \sum_{t=1}^{3} u p e_{p t}^{ \pm} \times \sum_{i=1}^{8} s a e_{p i t}^{ \pm}+\sum_{s=1}^{4} \sum_{t=1}^{3} u p e_{s t}^{ \pm} \times \sum_{i=1}^{8} s a e_{s i t}^{ \pm}
$$

Where $p$ is primary energy type, $p=1$ coal, $p=2$ oil, $p=3$ natural gas; $s$ is secondary energy type, $s=1$ coke, $s$ $=2$ coke oven gas, $s=3$ electricity, $s=3$ coal gangue; $i$ is industrial type, $i=1$ coal mining and washing industry, $i$ $=2$ oil and gas industry, $i=3$ petroleum processing, coking, industry, $i=4$ electricity and heat production and supply 
industry, $i=5$ chemical raw materials and chemical manufacturing industry, $i=6$ non-metallic mineral products industry, $i=7$ ferrous metal smelting and rolling processing industry, $i=8$ non-ferrous metal smelting and rolling processing industry; $t$ is time period, $t=12021$ 2025, $t=2$ 2026-2030, $t=3$ 2031-2035; upe $e_{p t}^{ \pm}$and $u p e_{s t}^{ \pm}$respectively are unit-prices of $p$ and $s$ energy in $t$ period; $s a e_{p i t}^{ \pm}$and $s a e_{\text {sit }}^{ \pm}$respectively are supply amounts of $p$ and $s$ energy for $i$ industry in $t$ period.

(2) Energy transportation cost:

$C_{Y}=\sum_{p=1}^{3} \sum_{t=1}^{3} t c e_{p t}^{ \pm} \times\left(1+u p e_{p t}^{ \pm}\right) \times \sum_{i=1}^{8} s a e_{p i t}^{ \pm}+\sum_{s=1}^{4} \sum_{t=1}^{3} t c e_{s t}^{ \pm} \times\left(1+u p e_{s t}^{ \pm}\right) \times \sum_{i=1}^{8} s a e_{s i t}^{ \pm}$

Where $t c e_{p t}^{ \pm}$and $t c e_{s t}^{ \pm}$respectively are unit-prices of $p$ and $s$ energy in $t$ period.

(3) Industrial production cost:

$$
C_{S}=\sum_{p=1}^{3} \sum_{t=1}^{3} m p e_{p t}^{ \pm} \times p p e_{p t}^{ \pm}+\sum_{s=1}^{4} \sum_{t=1}^{3} m p e_{s t}^{ \pm} \times p p e_{s t}^{ \pm}+\sum_{i=1}^{8} \sum_{t=1}^{3} i p o_{i t}^{ \pm} \times i p p_{i t}^{ \pm}
$$

Where $m p e_{p t}^{ \pm}$and $m p e_{s t}^{ \pm}$respectively are total outputs of $p$ and $s$ energy in $t$ period; $p p e_{p t}^{ \pm}$and $p p e_{s t}^{ \pm}$ respectively are mining and processing costs of unit $p$ and $s$ energy in $t$ period; ipo $o_{i t}^{ \pm}$are production costs of unit $i$ industry product in $t$ period; ipp $p_{i t}^{ \pm}$are product outputs of $i$ industry in $t$ period.

(4) Pollutant treatment cost:

$$
C_{W}=\sum_{t=1}^{3} \sum_{w=1}^{2}\left(\sum_{p=1}^{3} p e m_{p w t}^{ \pm} \times \sum_{i=1}^{8} s a e_{p i t}^{ \pm}+\sum_{s=1}^{4} p e m_{s w t}^{ \pm} \times \sum_{i=1}^{8} s a e_{p i t}^{ \pm}\right) \times p u d_{w t}^{ \pm}+p t e_{i t}^{ \pm}
$$

Where $w$ is pollutant type, $w=1 \mathrm{SO}_{2}, w=2 \mathrm{NO}_{2}$; pem $_{p w t}^{ \pm}$and pem $_{s w t}^{ \pm}$respectively are emission coefficients of $p$ and $s$ energy to $w$ pollutant in $t$ period; $p u d_{w t}^{ \pm}$are disposal costs of unit $w$ pollutant in $t$ period; $p t e_{i t}^{ \pm}$are purchase costs of pollutant treatment equipment of $i$ industry in $t$ period.

(5) Pollutant discharge cost:

$$
C_{W}=\sum_{t=1}^{3} \sum_{w=1}^{2}\left(\sum_{p=1}^{3} p e m_{p w t}^{ \pm} \times \sum_{i=1}^{8} s a e_{p i t}^{ \pm}+\sum_{s=1}^{4} p e m_{s w t}^{ \pm} \times \sum_{i=1}^{8} s a e_{p i t}^{ \pm}\right) \times\left(1-p e t_{w}\right) \times p d c_{w} / p v e_{w}
$$

Where $p e t_{w}^{ \pm}, p d c_{w}^{ \pm}$and $p v e_{w}^{ \pm}$respectively are treatment efficiencies, discharge costs and equivalent values of $w$ pollutant.

\subsubsection{Constrains. The constraints of CSIE model are formulated as follows:}

(1) Energy availability constraints:

Table 2. Representative values of different scenarios based on Clayton-Copula

\begin{tabular}{|c|c|c|c|c|c|}
\hline Scenarios & $H_{X, Y}(x, y)$ & $H_{Y \mid X}(y \mid X \leq x)$ & $F_{X}(x)$ & $G_{Y}(y)$ & $\left(\mathrm{B}, \beta_{1}, \beta_{2}\right)$ \\
\hline $\mathrm{C} 1$ & 0.900 & 0.877 & 0.920 & 0.877 & $(0.100,0.080,0.123)$ \\
\hline $\mathrm{C} 2$ & 0.900 & 0.885 & 0.940 & 0.885 & $(0.100,0.060,0.115)$ \\
\hline $\mathrm{C} 3$ & 0.900 & 0.891 & 0.960 & 0.891 & $(0.100,0.040,0.109)$ \\
\hline
\end{tabular}

$$
\begin{gathered}
\operatorname{Pr}\left\{\sum_{p=1}^{3} \sum_{t=1}^{3}\left(\sum_{i=1}^{8} s a e_{p i t}^{ \pm}\right) \leq m p e_{p t}^{ \pm}\right\} \geq 1-\beta_{1} \\
\operatorname{Pr}\left\{\sum_{s=1}^{4} \sum_{t=1}^{3}\left(\sum_{i=1}^{8} s a e_{s i t}^{ \pm}\right) \leq m p e_{s t}^{ \pm}\right\} \geq 1-\beta_{2} \\
C\left(1-\beta_{1}, 1-\beta_{2}\right)=1-\mathrm{B}
\end{gathered}
$$

Where $\beta_{1}$ and $\beta_{2}$ respectively are individual constraint-violations corresponding to $p$ and $s$ energy output; B are prescribed joint constraint-violations.

(2) Industrial product demand constraints:

$$
\sum_{i=1}^{8} \sum_{t=1}^{3} i p p_{i t}^{ \pm} \geq i p d_{i t}^{ \pm}
$$

Where $i p d_{i t}^{ \pm}$are product demands of $i$ industry in $t$ period.

(3) Environmental requirement constraints. Gaussian dispersion model is introduced to describe the concentration of pollutants.

$$
\sum_{i=1}^{8} \frac{1}{\pi \bar{u} \sigma_{y} \sigma_{z}} \exp \left(-\frac{H^{2}}{2 \sigma_{z}^{2}}\right) \times p e r_{w i t}^{ \pm} \times\left(1-\text { pet }_{w}\right) \leq p c s_{w t}
$$

Where $\operatorname{per}_{w i t}^{ \pm}$are pollutant emission rates of $w$ pollutant for $i$ industry in $t$ period; $p c s_{w t}$ are pollutant concentration standards of $w$ pollutant in $t$ period; $\sigma_{y}$ and $\sigma_{z}$ respectively are standard deviations of plume at $y$ and $z$ direction; $\bar{u}$ is wind velocity; $H$ is average effective stack height.

(4) Nonnegative constrains:

$$
\operatorname{sae}_{\text {pit }}^{ \pm}, \operatorname{sae}_{\text {sit }}^{ \pm}, i p p_{i t}^{ \pm} \geq 0
$$

\subsubsection{Scenario definition.}

In this study, primary energy output (X) and secondary energy output (Y) are two interrelated random variables, and their joint cumulative distribution function was calculated by Clayton-Copula. Two joint probability levels of 0.900 and 0.950 with three sets of individual probability levels at each joint probability level were considered to examine energy demand-supply risks. Table 2 provides representative values of different scenarios based on Clayton-Copula, where $H_{X, Y}(x, y)$ is joint cumulative distribution, $H_{Y \mid X}(y \mid X \leqslant x)$ is conditional cumulative distribution, $F_{X}(x)$ and $G_{Y}(y)$ respectively are marginal cumulative distribution, $\mathrm{B}$ is joint constraintviolation level, $\beta_{1}$ and $\beta_{2}$ are individual constraintviolation levels corresponding to $X$ and $Y$, respectively. 


\begin{tabular}{|l|l|l|l|l|l|}
\hline C4 & 0.950 & 0.930 & 0.960 & 0.930 & $(0.050,0.040,0.070)$ \\
\hline C5 & 0.950 & 0.936 & 0.970 & 0.936 & $(0.050,0.030,0.064)$ \\
\hline C6 & 0.950 & 0.941 & 0.980 & 0.941 & $(0.050,0.020,0.059)$ \\
\hline
\end{tabular}

\section{Result Analysis}

Industrial energy demand-supply risks of Yulin city during 2021-2035 were examined through three sets of individual constraint-violation levels at joint constraint-violation levels of 0.100 and 0.050 . Figure 2 illustrates industrial energy consumption scheme and the corresponding pollutant emission under different scenarios. It can be seen from the figure that a lower joint constraint-violation level would lead to a higher industrial energy consumption and pollutant emission. This is because a lower joint constraint-violation level indicates a higher energy output, corresponding to an increase in industrial energy consumption, and it would further lead to a higher pollutant emission; however, the system would show a worse suitability to environmental policy changes. Conversely, a higher joint constraint-violation level indicates a lower energy output, corresponding to a decrease in industrial energy consumption, then resulting in a lower pollutant emission; nevertheless, the system would suffer higher risks of industrial energy shortage. Therefore, there is a tradeoff between industrial energy consumption and pollutant emission.
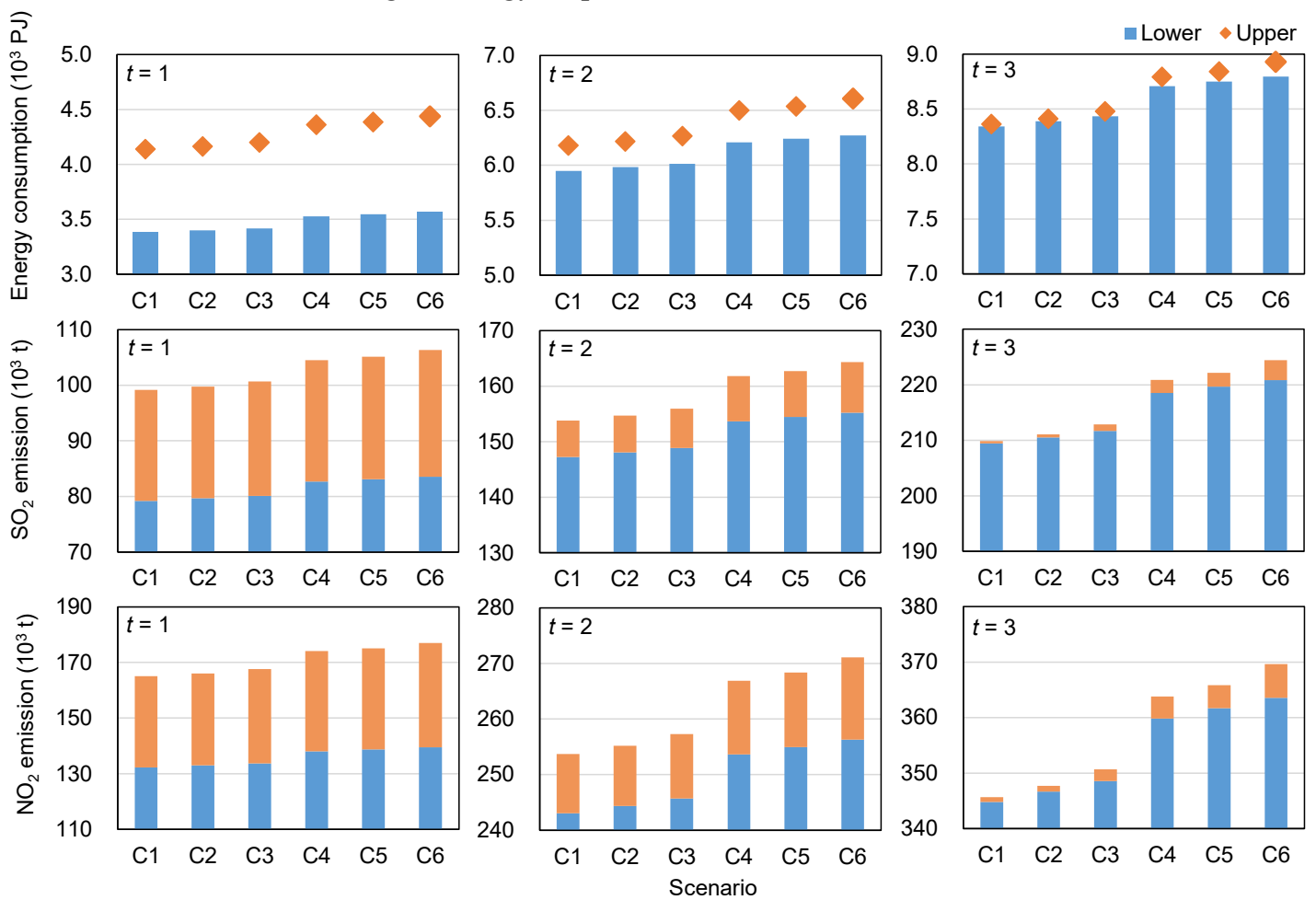

Figure 2. Energy consumption and pollutant emission under different scenarios

Figure 3 shows the system cost under different scenarios. The results disclosed that system cost would show an upward trend with the decreased joint constraintviolation levels and individual constraint-violation levels. Therefore, decision makers should adjust energy production and industrial energy consumption according to the actual environmental policy and industrial product demand. Specifically, decision makers should adopt a tight energy production policy if environmental requirements are strict, and vice versa. In this study, the system would have the lowest cost (i.e., RMB $¥[9.64,12.68] \times 10^{12}$ ) under scenario $\mathrm{C} 1$. Thus, the $\mathrm{C} 1$ would be selected as the best decision scheme. In detail, the system cost would be the lowest if and only if primary and secondary energy output are reduced by $8.0 \%$ and $12.3 \%$ respectively during 2021-2035.

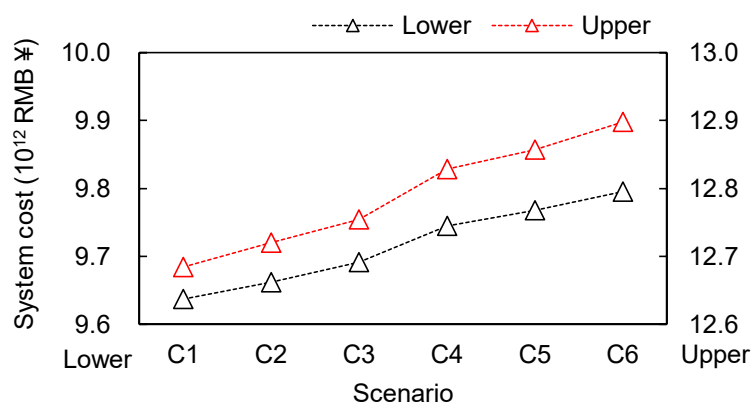

Figure 3. System cost under different scenarios 


\section{Conclusion}

In this study, a Copula-based stochastic industry-energy system management (CSIE) model was developed. CSIE model has advantages in extreme random uncertainties reflection, especially the uncertainties in random variables, which have previously unknown correlations. To verify the reliability of CSIE model, it was applied to a real case study of industry-energy system planning in Yulin city. The results show that the system cost would be the lowest during 2021-2035 when primary and secondary energy output increases by $8.0 \%$ and $12.3 \%$ respectively.

\section{Acknowledgments}

This research was supported by the National Key Research Projects (2016YFC0207800) and National Natural Science Foundation of China (51608422).

\section{References}

1. Wang, J.Y., Wang, S.J., Li, S.J., Feng, K.S. (2019) Coupling analysis of urbanization and energyenvironment efficiency: Evidence from Guangdong province. Appl. Energy, 254: 113650.

2. Zhu, Y., Yan, X.X., Kong, X.M., Tong, Q.L., Li, Y.P., Huang, G.H., Li, Y.X. (2019) Industry-environment system management based on an uncertain Gaussian diffusion optimization model for coal-dependent cities in ecologically fragile areas. J. Clean Prod., 234: 832-857.

3. Jin, S.W., Li, Y.P., Yu, L., Suo, C., Zhang, K. (2020) Multidivisional planning model for energy, water and environment considering synergies, trade-offs and uncertainty. J. Clean Prod., 259: 121070.

4. Baak, M., Koopman, R., Snoek, H., Klous, S. (2020) A new correlation coefficient between categorical, ordinal and interval variables with Pearson characteristics. Comput. Stat. Data. An., 152: 107043.

5. Fan, Y.R., Huang, W.W., Huang, G.H., Huang, K., Li, Y.P., Kong, X.M. (2015) Bivariate hydrologic risk analysis based on a coupled entropy-copula method for the Xiangxi River in the Three Gorges Reservoir area, China. Theor. Appl. Climatol., 125: 381-397. 